\title{
Review of Landscape Analysis and Visualisation; Spatial Models for Natural Resource Management and Planning, eds. Christopher Pettit, William Cartwright, Ian Bishop, Kim Lowell, David Pullar, David Duncan
}

\section{Publisher: Springer}

\section{Stan Geertman}

Received: 13 May 2009 / Accepted: 13 July 2009 /

Published online: 9 September 2009

C The Author(s) 2009. This article is published with open access at Springerlink.com

This book contains an impressive compilation of chapters concerning the landscape concept and the way in which analysis and visualisation tools can be of help in understanding, interpreting, and communicating such a multidimensional and complex spatial unity. The chapters originate from a conference (Place and Purpose-Spatial models for natural resource management and planning), held in 2007 in Bendigo, Victoria, Australia.

Some characteristics stand out and deserve some special attention. First, most of the contributions are written by Australian authors and/or focus on Australian landscapes. As stated by Mike Batty in his preface '... Australia — where landscapes dominate and their relationship to cities is so fragile, yet so important to the sustainability of an entire nation, if not planet.' This Australian flavour can hardly be envisioned a limitation, conversely it can be seen as a strong point given the dominant position of landscapes and landscape research within the Australian context.

Another characteristic concerns the wide diversity of topics the book handles. In fact, this is a logical outcome of the statement made by the editors in their introductory chapter that a landscape is a dynamic system of both living and nonliving objects, characterized by many dimensions. To be able to understand and manage landscapes in all their complexity asks for attention to both biophysical (flora and fauna), geomorphologic, social (anthropogenic) and economic (natural resource) dimensions at least. All these topics are dealt with in this book, which can be considered a huge effort.

An additional characteristic is the focus of the book which puts attention to the frameworks, models, and tools to analyse and visualize landscapes. The underlying idea is that to be able to achieve the goal of sustainability it is of prime importance to

\footnotetext{
S. Geertman $(\bowtie)$

Department of Human Geography and Urban and Regional Planning, University of Utrecht,

Utrecht, the Netherlands

e-mail: s.geertman@geo.uu.nl
} 
understand and manage landscapes in all their complexity. This requires knowledge of landscape dimensions and effective communication thereof to the wide diversity of involved participants such as decision makers, planners, resource managers, landholders and communities.

A further aspect of the book is the acknowledgement that to be able to grasp the complexity of the landscape concept, reference should be made not just to expert knowledge but also to local, tacit knowledge. Consequently, the bridging of differential knowledge categories is considered a complex but essential endeavour in order to achieve the goal of sustainability and in that can be envisioned a challenging characteristic of this book.

The book is compiled of five parts. In part 1 attention is focused on frameworks, models and tools for natural resource management. Increasingly natural resource management is becoming a holistic endeavour which challenges decision making substantially, not at least due to the encountered trade-offs. The chapters in this part offer insights into the different ways to handle complex trade-offs within natural resource management. Attention is focused on decision making frameworks (e.g., Carl Steinitz' landscape framework), on data frameworks needed (e.g., Spatial Data Infrastructures), on support tools like catchment analysis tools and spatial multicriteria analysis tools, and on methods to translate the results of science into policy implications (e.g., evidence-based policy).

In part 2 attention is shifting to the integration of biodiversity and ecological processes into landscape analysis and visualisation. Interesting themes and dilemmas are posed here, for example, the observation that 'the best' landscape biodiversity for humans is not automatically 'the best' for other species, which will have its influence on our concept of sustainable biodiversity to strive for, and as a consequence also on the best ways to analyse and visualize landscapes. Besides this kind of 'what' questions also the more procedural 'how' questions are posed, in which among other things a so-called 'adaptive management' paradigm is proposed.

In part 3 the socioeconomic dimensions of landscapes are at stake. It is generally acknowledged that social and behavioural sciences need to play a much more important role in understanding and management of landscapes. As a consequence, diverse conceptual frameworks are presented in which community and social values are incorporated in understanding landscape changes. Also a range of analytical and supporting methods and techniques are presented, for example, the Principal Component Analysis and a decision adding system; all intending to provide valuable insights concerning the landscape to policy making.

In part 4 the focus is shift to the field of land use modelling. Central to this is the goal that with the help of modelling tools new insights into land use change can be gained. Well-known models like SLEUTH and What-If? are presented briefly and additionally applied within diverse landscapes to build land use scenarios. Several key concepts of land use modelling are addressed like differential levels of analysis, detection of driving forces, and modelling of temporal dynamics.

In the concluding part 5 attention is moving again, now to the visualisation of landscapes. Quite a diversity of visualisation techniques is presented like virtual globes, computer games and GIS-based virtual worlds. Moreover, these techniques are put into practice in a wide diversity of application fields like rural landscape scenario building, urban fabrics, re-vegetation of saline landscapes, etc. etc. 
In reflection one has to acknowledge this book entails an impressive collection of contributions, impressive not just given the amount of pages (614 pages), but foremost in its diversity of topics and angles of incidence, all associated to the landscape concept. As stated in the beginning of this review the landscape concept is quite multidimensional and it presents a rather complex system, in which biotic and abiotic elements and systems interact at different time and spatial scales, resulting in a diverse range of landscape forms and contents. Studying its constituting elements and their-mutual and surrounding - interactions, and researching the ways in which the landscape can and/or will develop itself and how this all can be visualised for communication and decision support purposes, this all is included in this work. Therefore I consider this book of substantial value and indispensible for people interested in methods and techniques for landscape analysis and visualisation. This work will provide them with a very nice overview, dedicated to a broad public of academic and practitioners alike. As developments continue at a high speed, hopeand expect-other books dedicated to the field of landscape analysis and visualisation for management and planning will follow.

Open Access This article is distributed under the terms of the Creative Commons Attribution Noncommercial License which permits any noncommercial use, distribution, and reproduction in any medium, provided the original author(s) and source are credited. 